Article

\title{
Transcriptome Profiling of Haloxylon persicum (Bunge ex Boiss and Buhse) an Endangered Plant Species under PEG-Induced Drought Stress
}

\author{
Fayas Thayale Purayil ${ }^{1,2,+} \mathbb{C}^{\text {, Balaji Rajashekar }}{ }^{3,4,+}{ }^{\mathbb{C}}$, Shyam S. Kurup ${ }^{1, *}$, \\ Abdul Jaleel Cheruth ${ }^{1}$, Sreeramanan Subramaniam ${ }^{5}$, Nadia Hassan Tawfik ${ }^{1}$ \\ and Khaled M.A. Amiri $2,6, *$ (D) \\ 1 Department of Integrative Agriculture, College of Food and Agriculture, United Arab Emirates University, \\ P.O. Box. Al-Ain 15551, UAE; fayas.t@uaeu.ac.ae (F.T.P.); abdul.jaleel@uaeu.ac.ae (A.J.C.); \\ nadia.hassan@uaeu.ac.ae (N.H.T.) \\ 2 Khalifa Center for Genetic Engineering and Biotechnology, United Arab Emirates University, \\ P.O. Box. Al Ain 15551, UAE \\ 3 Institute of Computer Science, University of Tartu, 50409 Tartu, Estonia; balajior@gmail.com \\ 4 Celixa, Bangalore, Karnataka 560020, India \\ 5 School of Biological Sciences, Universiti Sains Malaysia (USM), Minden Heights, Georgetown, \\ Penang 11800, Malaysia; sreeramanan@gmail.com \\ 6 Department of Biology, College of Science, United Arab Emirates University, P.O. Box. Al Ain 15551, UAE \\ * Correspondence: skurup@uaeu.ac.ae (S.S.K.); k.amiri@uaeu.ac.ae (K.M.A.) \\ + Co-first author.
}

Received: 2 May 2020; Accepted: 5 June 2020; Published: 10 June 2020

check for updates

\begin{abstract}
Haloxylon persicum is an endangered western Asiatic desert plant species, which survives under extreme environmental conditions. In this study, we focused on transcriptome analysis of H. persicum to understand the molecular mechanisms associated with drought tolerance. Two different periods of polyethylene glycol (PEG)-induced drought stress ( $48 \mathrm{~h}$ and $72 \mathrm{~h}$ ) were imposed on $H$. persicum under in vitro conditions, which resulted in 18 million reads, subsequently assembled by de novo method with more than 8000 transcripts in each treatment. The N50 values were 1437, 1467, and 1524 for the control sample, $48 \mathrm{~h}$ samples, and $72 \mathrm{~h}$ samples, respectively. The gene ontology (GO) and Kyoto encyclopedia of genes and genomes (KEGG) pathway analysis resulted in enrichment of mitogen-activated protein kinase (MAPK) and plant hormone signal transduction pathways under PEG-induced drought conditions. The differential gene expression analysis (DGEs) revealed significant changes in the expression pattern between the control and the treated samples. The KEGG analysis resulted in mapping transcripts with 138 different pathways reported in plants. The differential expression of drought-responsive transcription factors depicts the possible signaling cascades involved in drought tolerance. The present study provides greater insight into the fundamental transcriptome reprogramming of desert plants under drought.
\end{abstract}

Keywords: Haloxylon persicum; white saxaul; drought resistant; de novo transcriptome

\section{Introduction}

H. persicum (Bunge ex Boiss and Buhse), the white saxaul belongs to the family Amaranthaceae, is an extremely drought-tolerant tree species with sand dunes as the habitat. It grows up to a height of $4 \mathrm{~m}$. In the United Arab Emirates (UAE), H. persicum is generally found in gravel plains and sand dunes and has a great potential in landscaping. The tree is evergreen in the habitat with the leaves retrogressed as succulent branches of the tree. The plant has an extensive root system, making it useful for stabilizing 
sandy soils, especially in the desert ecosystem. The trees cover a desert land area of $1 \mathrm{million}^{\mathrm{km}}{ }^{2}$ across the Turanian deserts and appears in the hot deserts of the Middle East. This species is scattered throughout northwestern China and northern Russia [1]. Zohary, (1973) reported that in the Middle East the species is distributed in the Sinai Peninsula, Egypt, and central and northwestern Saudi Arabia on the Arabian Peninsula [2]. It has tremendous potential to be used as a landscape plant for urban landscaping and afforestation programs in the desert dunes of Central Asia, for combating desertification and land degradation in arid regions in respect to drought and thermal combating ability. Thus, the plant has been screened for landscape qualities, especially on its evergreen nature, compact growing form, and branching character, which produce a high canopy density. These trees are fast becoming endangered in respect to many factors, including climate change, unmanaged and over-grazing, faster urbanization, and other anthropogenic factors. As a xerophytic desert tree, $H$. persicum has tremendous drought and thermal tolerance, and thus serves a very crucial role in maintaining the ecosystem and the habitat in which it grows. These woodlands need to be conserved, since this is the only naturally occurring stand of the species in the United Arab Emirates and in eastern Arabia in general. The species has medicinal and economic importance as well. These woodlands are also microhabitats for many species of birds and small mammals, who require its fortification for survival (Figure 1).

In arid regions, drought is one of the major environmental restrictions that limits growth and productivity. During drought stress the plants undergo several changes, which eventually lead to the reduction in gas exchange and photosynthesis level and a decrease in cell division and cell expansion due to reduced enzyme activities and a lack of energy [3,4]. To adapt to these conditions, plants undergo various physiological, molecular, and cellular modifications to acclimatize with the changes imposed during the drought stress. These complex mechanisms of drought tolerance show variations among different plant species [5]. Functional proteins and regulatory proteins are two major groups of stress-inducible proteins, which play an important role in plant adaptation to stress conditions. Functional proteins are directly involved in plant defense under stress, which includes genes that are involved in the biosynthesis of osmo-protectants, membrane proteins, genes encoding chaperons, genes involved in growth and development, and genes encoding detoxification enzymes, etc. Regulatory proteins are involved in the regulation of downstream genes in a stress-response pathway. This includes different types of proteins kinases and transcription factors [6-8].

In plants, the abscisic acid-mediated signaling pathway plays a central role in plant stress responses [9,10]. Under a water deficit condition, the accumulation of abscisic acid (ABA) levels trigger the expression of ABA-responsive genes, leading to changes in physiological responses such as stomatal closure and other developmental process to alleviate the stress situation [11]. Under stress conditions, the regulation of stress-responsive genes is controlled by various transcription factors such as the bZIP family, the AP2/ERF superfamily, and the NAC family, etc. These transcription factors have significant roles in plant defense through ABA-dependent and ABA-independent stress-tolerance mechanisms [11,12]. Mitogen-activated protein kinase (MAPK) cascade is one of the major abiotic stress-response pathways involved in transducing external stimuli to the nucleus for appropriate adjustment of cellular responses under stress [13]. The role of MAPK-signaling cascade in abiotic stress, such as salt, osmotic stress, drought, heat, and cold, has been elucidated in different plant species [14]. Under stress condition, the phosphorylation of target genes are regulated by MAPK, which controls the activity of different structural proteins and various transcription factors which are involved in abiotic stress tolerance. Along with transcription factors (TFs), MAPK signal cascade also plays an important role in ABA-dependent and ABA-independent abiotic stress responses in plants [15]. Overall, various metabolic pathways and signaling molecules are involved in plants defense against abiotic stress. A balanced homeostasis of genes involved in various metabolic pathways are important for plant growth and development under stress [16].

Understanding the drought-tolerance mechanism is one of the critical steps towards the conservation and improvement of genetic resources for future. With the advent of next generation 
sequencing (NGS), efficient technologies are available for identifying the gene regulatory mechanism in plants. Deep ribonucleic acid (RNA) sequencing studies are achievable for all plant species by exploring transcriptome profiles of model and non-model organisms, under various conditions. These high-throughput sequencing techniques are widely used to create transcriptome data, which enables researchers to identify the differential expression profile of genes in plants under different treatment conditions [17-19]. In recent times, transcriptomic approaches have gained much attention for identifying the gene regulatory network associated with drought-stress tolerance in plants [20,21]. In this study, polyethylene glycol (PEG) 6000 (10\%) was supplemented in culture medium to induce drought stress in $H$. persicum under in vitro conditions. Understanding the transcriptome profile under PEG-induced drought stress will enable us to identify the differential expression profile of genes under stress. Although some differences may occur between the responses to PEG-induced drought and the drought under field conditions, the present study will evidently provide greater insight into the regulatory networks associated with drought tolerance in these xerophytic plant species that can adapt to harsh climates of the arid region.

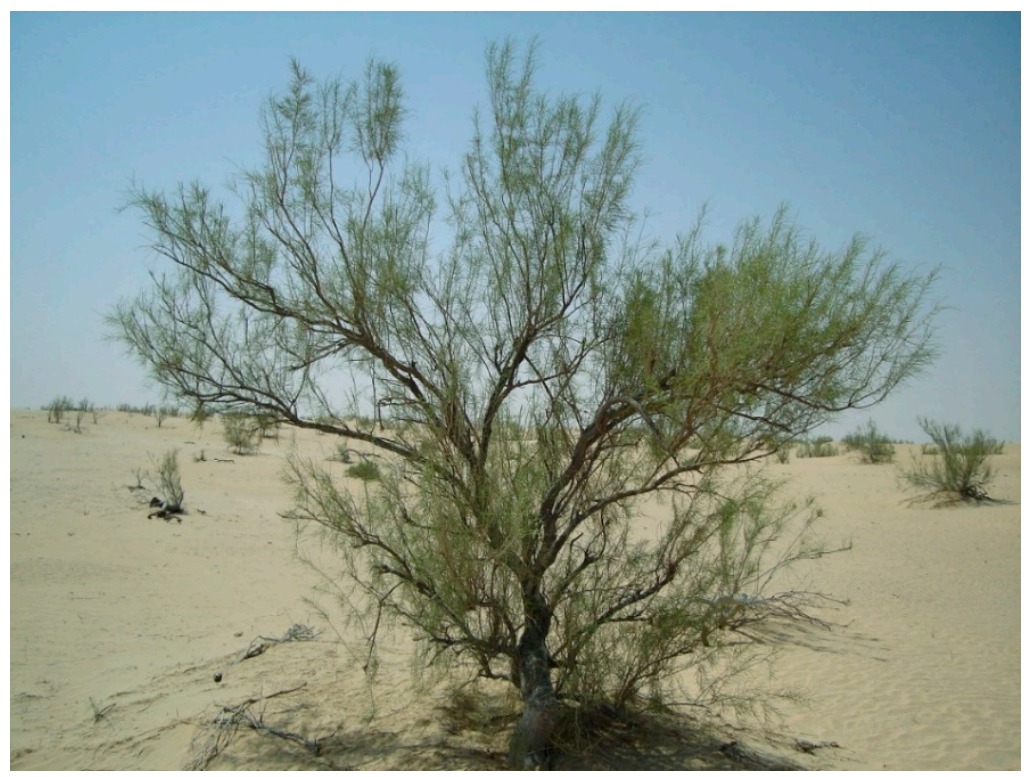

Figure 1. The H. persicum plant in its natural habitat, sand dunes.

\section{Materials and Methods}

\subsection{Plant Material and Drought Treatment}

Two-year-old seedlings of $H$. persicum maintained in the greenhouse conditions, used for the study were provided by Environmental Agency, Abu Dhabi (UAE). Apical shoot bud explants collected from the seedlings were surface sterilized by washing initially with running tap water and then with $1 \%$ Tween 20 solution to remove any adhering contaminants. Under sterile conditions, the explants were treated with $20 \%$ sodium hypochlorite for $10 \mathrm{~min}$ and washed $4-5$ times with sterile distilled water. The sterilized explants were inoculated onto a Murashige and Skoog (MS) medium and incubated at $25 \pm 2{ }^{\circ} \mathrm{C}$ under $16 \mathrm{~h}$ photoperiod (3678 Lux) and $8 \mathrm{~h}$ dark conditions.

The one-month-old adventitious shoot buds of $H$. persicum cultures were treated with PEG $600010 \%$ (-0.60 MPa) in MS media for drought induction under in vitro conditions. The shoot buds were maintained at $16 \mathrm{~h}$ photoperiod and $8 \mathrm{~h}$ dark conditions at $25 \pm 2{ }^{\circ} \mathrm{C}$. Samples were collected at $48 \mathrm{~h}$ and $72 \mathrm{~h}$ of treatment under PEG media. The MS medium without PEG (-3.2 MPa) served as the control. Each treatment had three replicates and the samples were collected after the preferred incubation period. The water potential of the drought-treated and control samples were measured 
using a WP4C water potential meter (Meter, Pullman, WA, USA). The relative water content (RWC) was measured using the following equation [22]:

$$
R W C=[(F W-D W) \div(T W-D W)] \times 100
$$

where, $F W$ is fresh weight, $D W$ is dry weight and $T W$ is turgid weight. $D W$ is calculated after drying the samples for $24 \mathrm{~h}$ at $80{ }^{\circ} \mathrm{C}$.

\subsection{RNA Isolation and Transcriptome Library Preparation}

The samples were collected from the PEG treatment at different periods of drought induction for RNA isolation and transcriptome profiling. The purification of RNA was carried out using a RNeasy mini kit (Qiagen, Germantown, MD, USA). The RNA concentration and purity were estimated using Nanodrop spectrophotometer (Thermo Fisher Scientific, Wilmington, DE, USA). The RNA integrity of the samples was checked using a Bioanalyzer (Agilent Technologies, Santa Clara, CA, USA). Briefly, $200 \mathrm{ng}$ of RNA was used for mRNA isolation, fragmentation and priming. The first strand cDNA was synthesized in the presence of Actinomycin D, followed by second strand synthesis. The double stranded cDNA was purified using HighPrep PCR magnetic beads (Magbio Genomics Inc., Gaithersburg, MD, USA). Three replicates of each samples were pooled to form a single RNA sequencing library. The library was prepared with Illumina-compatible NEBNext ${ }^{\circledR}$ UltraTM Directional RNA Library Prep Kit (New England BioLabs, Ipswich, MA, USA) based on the manufacturer's instructions. Transcriptome sequencing was carried out using Illumina HiSEQ2500 at Genotypic Technology (Bangalore, India). The purified RNA was stored at $-80{ }^{\circ} \mathrm{C}$ for real-time fluorescent quantitative PCR validation.

\subsection{Illumina Raw Data Processing and Transcriptome Assembly}

The raw illumina paired end (PE) read quality was assessed using FastQC tool [23]. Reads were trimmed by removing $3^{\prime}$ adapter, trimming ambiguous bases towards the read end and removal of PE reads, which had more than $30 \%$ of low quality $(<q 30)$ bases. Reads over 50 bp in length were retained after trimming process. Transcriptome de novo assembly was carried out using Trinity software, with the default k-mer value of 25 [24]. A Linux-based server with 256 GB of RAM and 20 cores was used for the transcriptome assembly. The redundant transcripts were clustered using CD-HIT program [25] to reduce redundancy and to obtain unigenes with sequence similarity (90\%) and identity $(95 \%)$ between the assembled transcripts. The clustered transcripts were used for further annotation with a minimum length of $300 \mathrm{bp}$.

\subsection{Transcriptome Functional Annotation}

The transcripts were annotated with the BlastX program (significant e-value of $6.738 \times 10^{-3}$ ) [25] against NCBI Uniprot Viridiplantae sequences. The gene ontology information (Biological Process, Molecular Function and Cellular Component) associated with the transcripts was identified using the Blast2GO program [26]. Further Clusters of Orthologous Groups (COGs) classification was carried out using the NCBI-COG database [27]. Using the KEGG Automatic Annotation Server (KAAS) tool [28], transcripts were searched against the Kyoto encyclopedia of genes and genomes database (KEGG) [29] and KEGG orthology for identifying the metabolic pathways. During the pathway analysis, Arabidopsis thaliana (thale cress), Tarenaya hassleriana (spider flower), Fragaria vesca (woodland strawberry), Theobroma cacao (cacao), Populus trichocarpa (black cottonwood), Glycine max (soybean), Citrus sinensis (Valencia orange), Cucumis sativus (cucumber), Vitis vinifera (wine grape), Solanum lycopersicum (tomato) and Oryza sativa japonica (Japanese rice) were considered as reference organisms. Simple sequence repeats (SSRs) found in the clustered transcripts were identified using the MISA (http://pgrc.ipk-gatersleben.de/misa/) program, using the default settings. 


\subsection{Differential Gene Expression (DGE) Analysis}

The fragments per kilobase per million fragments (FPKM) approach was used to calculate the gene expression. Transcripts from all the samples (control, T1 and T2) were pooled and clustered based on the similarity between transcripts using CD-HIT. The reads from different samples were aligned to the clustered transcriptome using the bowtie2 program [30], a pair of reads which supported each transcript were counted, and the significant gene expression difference was calculated using the DESeq tool [31] (FDR less than 0.01 were considered as a significant result). A heat map for differential expression was generated using the R program (https://www.r-project.org/).

\section{6. qPCR Validation}

The RNA-sequence differential gene expression was confirmed using qPCR. Ten genes that showed the significant differential expression during the DGE analysis were used for qPCR confirmation. The total RNA was converted into cDNA using an Affinity Script qPCR cDNA synthesis kit (Agilent Technologies, Santa Clara, CA, USA) as per the manufacturer's protocol. In brief, $250 \mathrm{ng}$ of RNA from each sample was taken for cDNA synthesis and the first strand cDNA was synthesized using universal oligo dT primers. The cDNA was diluted to $20 \mathrm{ng} / \mu \mathrm{L}$ and $1 \mu \mathrm{L}$ of was used for each qPCR reaction. Specific qPCR primers were designed using the Primer3plus program (Supplementary Table S1). The expression levels of selected genes were analyzed using SYBR Green chemistry (Brilliant II SYBR Green qPCR master mix (Agilent Technologies, Santa Clara, CA, USA)) in Stratagene mx3005P instrument (Agilent Technologies, Santa Clara, CA, USA). The amplification cycling conditions were as follows: initial denaturation for $95^{\circ} \mathrm{C}$ for $10 \mathrm{~min}$, followed by 40 cycles of $95^{\circ} \mathrm{C}$ for $30 \mathrm{~s}, 60^{\circ} \mathrm{C}$ for $30 \mathrm{~s}$. The dissociation curve analysis was performed after amplification for primer specificity; the conditions were as follows: $95{ }^{\circ} \mathrm{C}$ for $1 \mathrm{~min}, 55^{\circ} \mathrm{C}$ for $30 \mathrm{~s}$ and $0.2{ }^{\circ} \mathrm{C} / \mathrm{s}$ increment up to $95^{\circ} \mathrm{C}$ (continuously collect fluorescence from $55-95^{\circ} \mathrm{C}$ ). Three replicates were included for each sample and the mean $\mathrm{Ct}$ value of the technical replicates was used to calculate the expression level. The relative quantification method $\left(2^{\hat{-}}-\Delta \Delta \mathrm{Ct}\right)$ was used to calculate the fold change using GAPDH as an internal control. The statistical significance between the treated samples were conducted by Student's t-test using SPSS 16.0.

\section{Results}

\subsection{Physiological Changes in H. persicum}

In the present study, in vitro drought stress studies in $\mathrm{H}$. persicum was conducted by using $10 \%$ PEG 6000 (Figure 2a). Physiological parameters, such as fresh weight, dry weight, tissue water potential, and relative water content, were measured in all the treatment samples. The fresh weight and dry weight of the tissues showed significant changes under PEG treatment. There was a significant decline in fresh weight and dry weight observed when the shoot buds were subjected to PEG stress for 48 and $72 \mathrm{~h}$. The fresh weight of the sample under control treatment was $0.505 \mathrm{~g}$, which reduced significantly to 0.455 and $0.430 \mathrm{~g}$ in samples that are cultured on PEG medium for $48 \mathrm{~h}$ and $72 \mathrm{~h}$, respectively. The dry weight of the tissues also showed gradual reduction upon increasing the PEG treatment period. The dry weight was $0.05 \mathrm{~g}$ under control condition, which reduced significantly to 0.0426 and $0.0353 \mathrm{~g}$ in tissues treated with PEG for $48 \mathrm{~h}$ and $72 \mathrm{~h}$ respectively (Figure $2 \mathrm{~b}$ ).

Relative water content and the tissue water potential $(\mathrm{MPa})$ were monitored to confirm the influence of PEG treatment in imposing drought under in vitro condition. The relative water content of the samples showed significant changes under PEG treatment. While a maximum of $90 \%$ moisture content was observed in the control samples, under PEG treatments the relative water content was significantly reduced to $84 \%$ after $48 \mathrm{~h}$. Due to the continued incubation of the cultures in the PEG treatment, the water content declined significantly to $81 \%$ after $72 \mathrm{~h}$ (Figure 2c). PEG 6000 treatment induced a significant reduction in tissue water potential. While the control samples without PEG 
showed $-0.25 \mathrm{MPa}$ water potential, after $48 \mathrm{~h}$ and $72 \mathrm{~h}$ of PEG treatment the tissue water potential significantly dropped to $-0.55 \mathrm{MPa}$ and $-0.91 \mathrm{MPa}$, respectively.

(a)

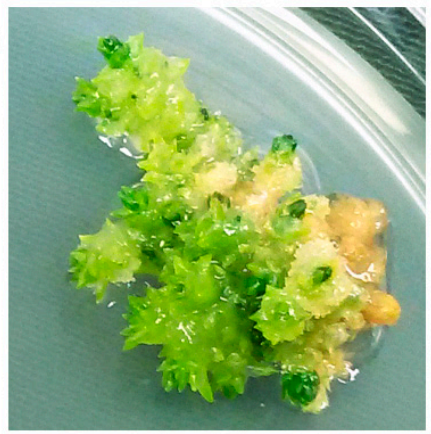

Control

(b)

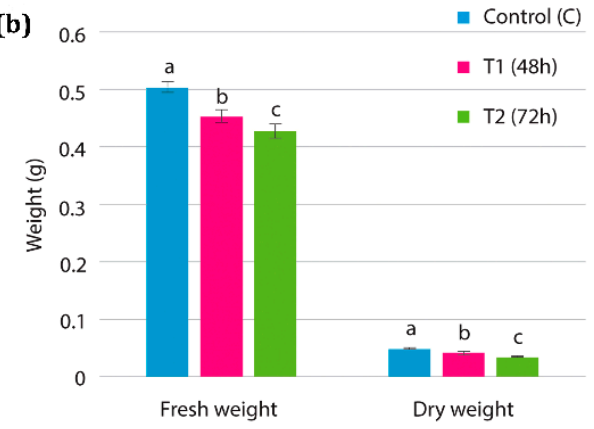

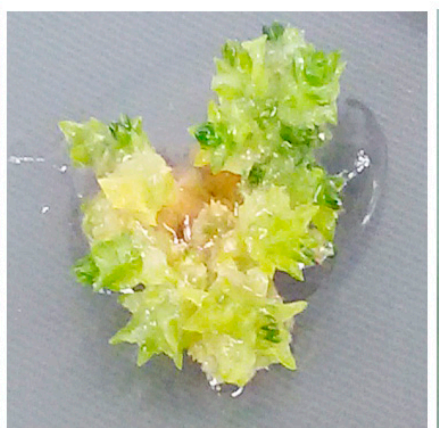

T1

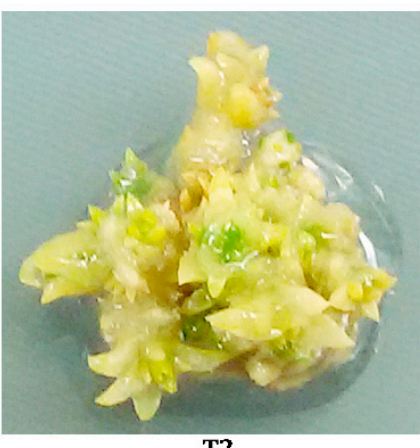

T2

Figure 2. The effect of polyethylene glycol (PEG)-induced drought stress in H. persicum. (a) PEG-induced drought on adventitious shoot buds under in vitro conditions. Control: shoot buds at control media (MS without PEG), T1: cultures at $48 \mathrm{~h}$ of PEG treatment, T2: cultures at $72 \mathrm{~h}$ of PEG treatment. (b) The effect of PEG treatment on tissue fresh weight $(\mathrm{g})$ and dry weight $(\mathrm{g})$ in control sample $(\mathrm{C})$, T1 sample (48 h PEG treatment) and T2 sample (72 h PEG treatment). (c) The effect of PEG treatment on Relative Water Content (RWC) in control samples, $48 \mathrm{~h}$ PEG treated samples and $72 \mathrm{~h}$ PEG treated samples. The error bar represents the standard deviation. Bar graph with different letters indicates significant difference between the mean values $(p \leq 0.05)$.

\subsection{Data Processing and Transcriptome Assembly}

The total RNA extracted from the control, T1, and T2 samples were converted into cDNA and sequenced using Illumina HiSeq2500 $(151 \times 2 \mathrm{bp}$ chemistry). The transcriptome sequencing of the control, T1, and T2 libraries generated 19, 22.4, and 21.7 million reads (Table 1). The sequenced raw reads were deposited in the NCBI-SRA database (control SAMN08281247, T1 sample SAMN08281244, and T2 sample SAMN08281248). Read trimming resulted in 15.2, 18 and 21.7 million high quality reads $(Q>30)$ for the control, T1, and T2 libraries, respectively. The length distribution of assembled transcriptome is shown in Figure 3 for the control, T1, and T2 sample, respectively. The adenine (A), thymine (T), guanine $(\mathrm{G})$ and cytosine $(\mathrm{C})$ content of each sample are listed in the Table 1 and Supplementary Figure S1a-c. The initial assembly of the control samples resulted in 110,448 contigs; these contigs were further clustered into 87,522 transcripts with a N50 value of $1437 \mathrm{bp}$ and GC percentage of 38.71, which represents $85.6 \mathrm{MB}$ of the control sample transcriptome. De novo assembly of both the T1 and T2 libraries resulted in 105,017 and 123,307 contigs; the clustering of these contigs generated 82,440 and 97,825 transcripts. The assembly for the T1 sample generated $81.3 \mathrm{MB}$ of transcriptome with the GC content of $38.92 \%$ and N50 value of $1467 \mathrm{bp}$. Similarly, the T2 sample generated $98.9 \mathrm{MB}$ of transcriptome with a N50 value and GC content of 1524 bp and 38.54\%, respectively (Table 1). 
Table 1. H. persicum transcriptome assembly statistics for the control sample, T1 (48 h PEG treatment) sample and T2 (72 h PEG treatment) sample.

\begin{tabular}{cccc}
\hline & Control & T1 & T2 \\
\hline Total raw reads (PE reads) & $19,007,972$ & $22,409,657$ & $21,771,386$ \\
\hline Total trimmed reads (PE reads) in Million & 15.24 & 18.04 & 17.12 \\
\hline Read length (bp) & 151 & 151 & 151 \\
\hline Total transcripts & 87522 & 82440 & 97825 \\
\hline Total bases & $85,611,629$ & $81,373,840$ & $98,916,209$ \\
\hline (G + C) \% & 38.71 & 38.92 & 38.54 \\
\hline N\% & 0 & 0 & 0 \\
\hline Minimum sequence length & 300 & 300 & 300 \\
\hline Maximum sequence length & 16,107 & 16,067 & 16,736 \\
\hline Average sequence length & 978.17 & 987.07 & 1011.15 \\
\hline N50 length & 1437 & 1467 & 1524 \\
\hline L50 number & 17,792 & 16,679 & 19,400 \\
\hline
\end{tabular}

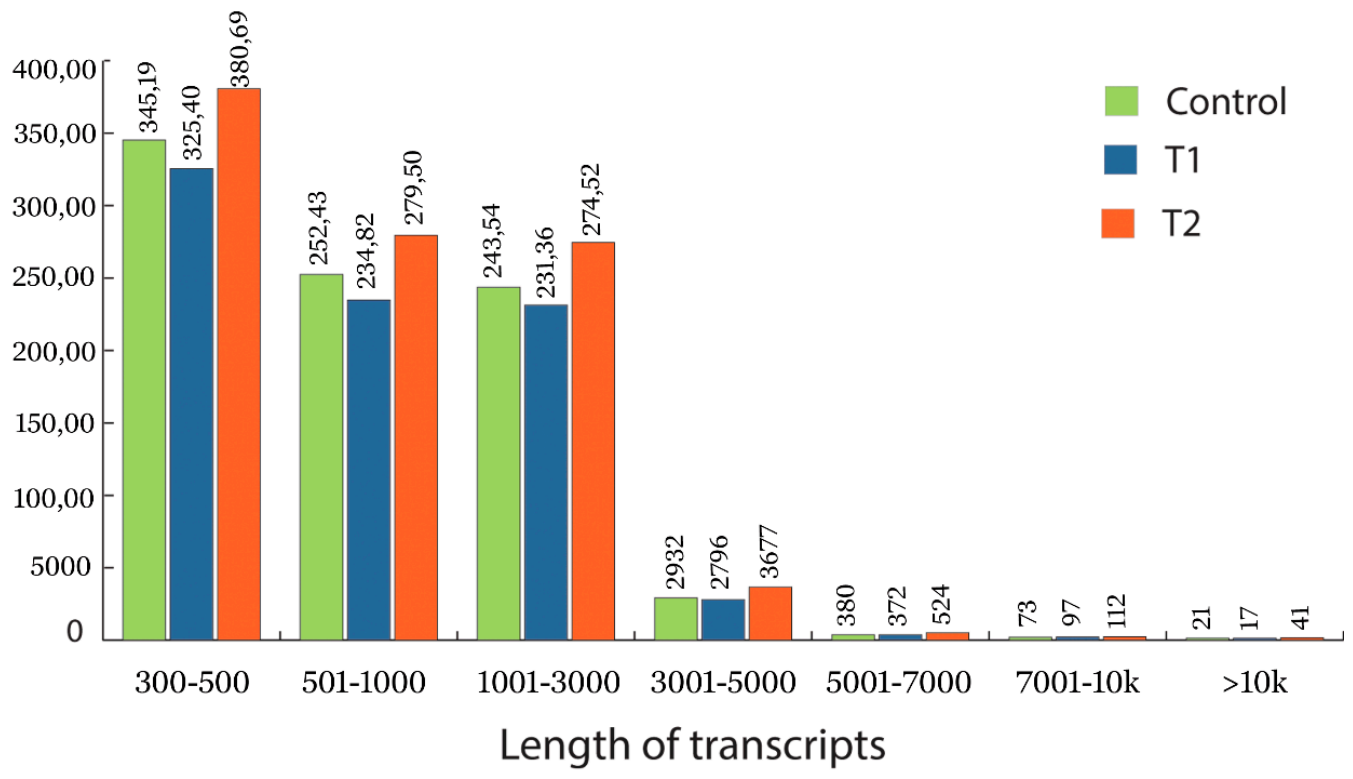

Figure 3. Length of the assembled transcript under different treatment conditions. Green represents the length distribution of the assembled transcriptome in the control samples of H. persicum, blue represents the length distribution of the assembled transcriptome in T1 (48 h PEG treatment), and red represents the length distribution of the assembled transcriptome in T2 (72 h PEG treatment).

\subsection{Transcriptome Annotation}

A similarity search against NCBI and Uniprot plant protein databases resulted in $53.6 \%$ (46,992 transcripts), $55.1 \%$ (45,468 transcripts), and 51.6\% (50,482 transcripts) of significant transcriptome annotations (e-value less than $6.738 \times 10^{-3}$ ) in the control, T1, and T2 samples, respectively (Supplementary Tables S2-S4). A major portion of the annotated transcripts shared high similarity with the Beta vulgaris subsp., Vulgaris ( 50\%), Spinacia oleracea $(\sim 35 \%)$ and other plants (Figure 4$)$. Further, the annotation resulted in the identification of important transcription factors that are involved in drought. The expression of the major transcription factors, such as NAC transcription factor (NAC), MYB family protein (MYB), dehydration-responsive element binding proteins (DREB), 
WRKY transcription factor family (WRKY), GATA transcription factors (GATA), and the basic leucine zipper domain (bZIP), were observed in all the samples sequenced.

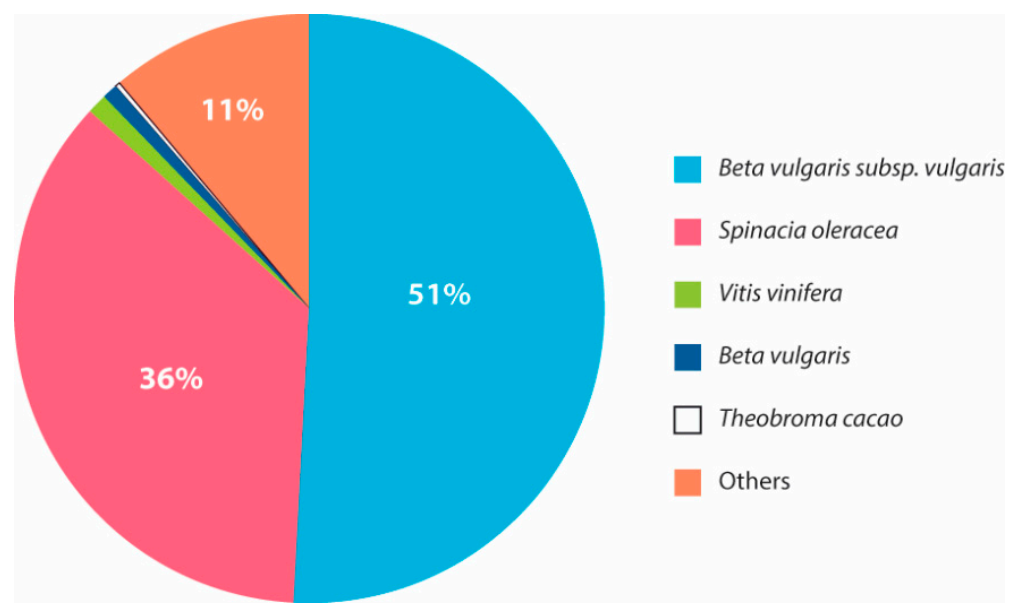

Figure 4. The percentage of similarity between the annotated $H$. persicum transcripts with other plant species.

\subsection{Gene Ontology (GO) Classification, SSR Mining and Pathway Analysis}

Gene ontology (GO) terms associated with each transcript were identified from Uniprot annotation based on the sequence homology. In the control sample, 26,253 Biological Process (BP), 24,148 Cellular Component (CC), and 44,004 Molecular Function (MF) related GO terms were identified (Figure 5a). In the T1 sample 25,490 BP, 23,494 CC, and 42,661 MF related GO terms were identified (Figure 5b). Similarly, in the T2 transcriptome 28,101 GO terms related to BP, 25,623 GO terms related to CC, and 46,873 MF related GO terms were identified (Figure 5c). Overall, the most expressed GO terms were GO:0016021 (integral component of membrane), GO:0005524 (ATP binding), GO:0005634 (nucleus), GO:0008270 (zinc ion binding), GO:0003676 (nucleic acid binding), GO:0003677 (DNA binding), GO:0046872 (metal ion binding), GO:0004672 (protein kinase activity), GO:0005737 (cytoplasm), GO:0005886 (plasma membrane), GO:0006355 (regulation of transcription, DNA-templated), and GO:0006351 (transcription, DNA-templated).

SSR mining was carried out using the MISA program. In total 19,548, 18,310, and 22,857 SSR repeats were identified in the control, T1, and T2 samples, respectively. From the overall samples, tetra repeats were found abundantly followed by tri and di repeats (Figure 6). The pathway analysis by KEGG-KAAS identified 138 plant-related metabolic pathways. In total, nearly 6200 transcripts functions were assigned to different pathways in all three samples. The major pathways identified in the transcriptome analysis were spliceosome(map03040), ribosome(map03010), carbon metabolism(map01200), protein processing in endoplasmic reticulum(map04141), plant-pathogen interaction(map04626), biosynthesis of amino acids(map01230), RNA transport(map03013), purine metabolism (map00230), plant-hormone signal transduction (map04075), mRNA surveillance pathway(map03015), endocytosis (map04144), pyrimidine metabolism (map00240), oxidative phosphorylation (map00190), RNA degradation (map03018), and ubiquitin-mediated proteolysis (map04120). 
(a)

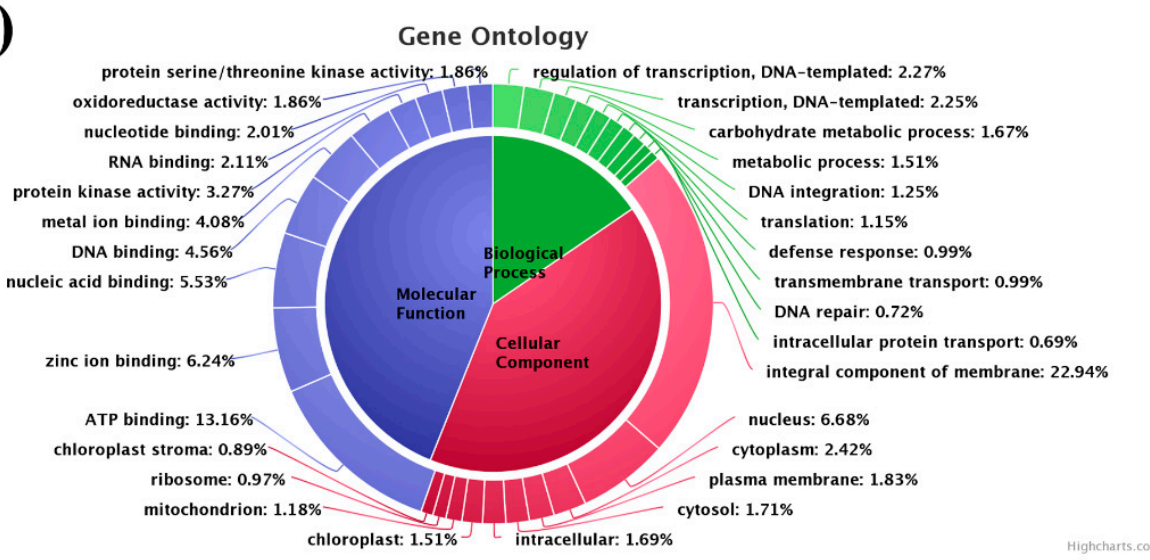

(b)

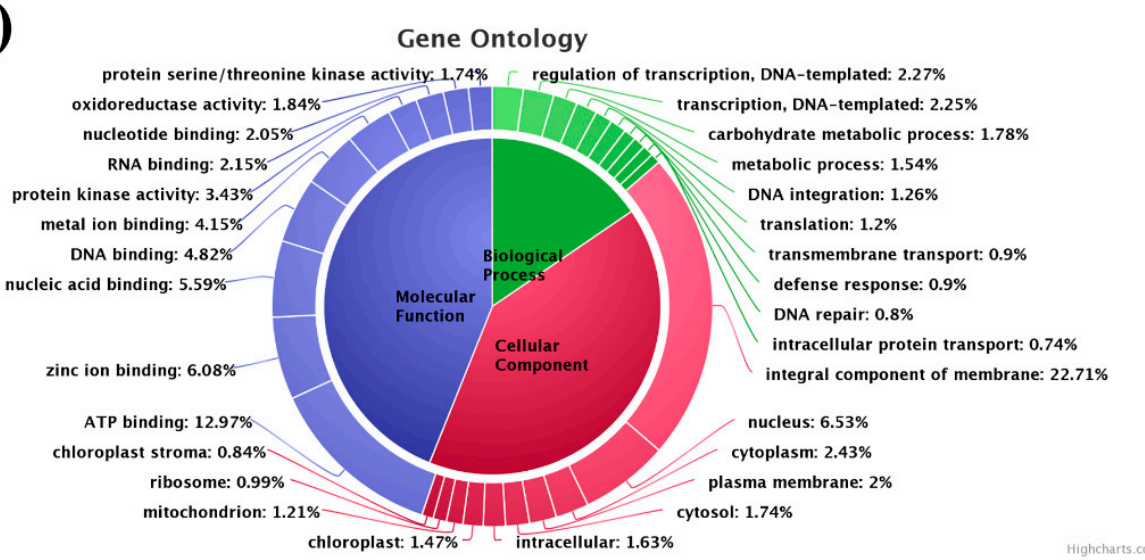

(c)

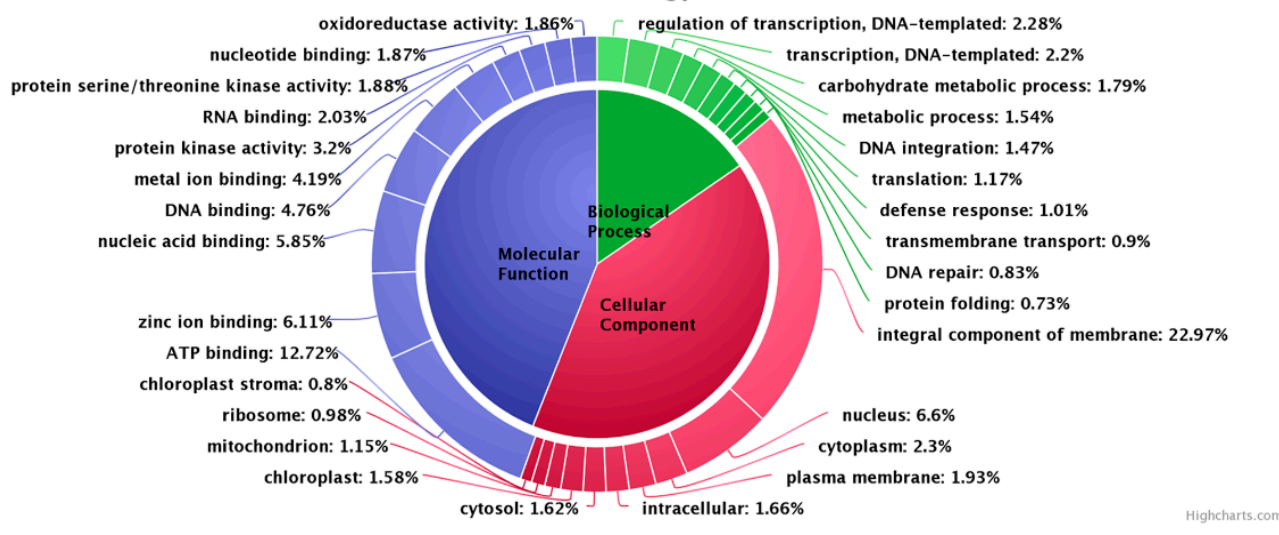

Figure 5. Gene ontology (GO) classification of the analyzed samples: (a) GO annotation in the control sample; (b) GO annotation in the T1 (48 h PEG treatment) sample; (c) GO annotation in the T2 (72 h PEG treatment) sample. 


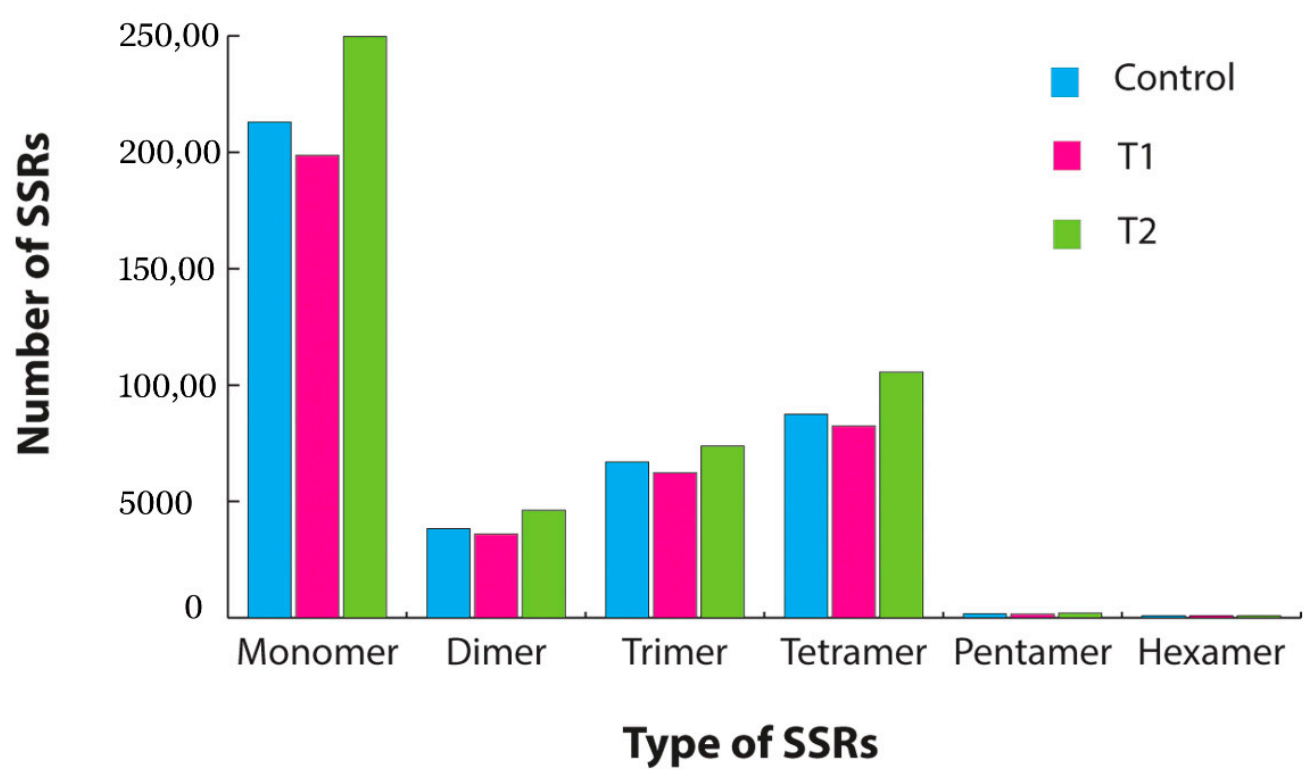

Figure 6. Simple sequence repeat (SSR) identification from the transcriptome data for each sample. Blue represents the identified SSR distribution in the control sample, pink represents the SSR distribution in the T1 (48 h PEG treatment) sample, and green represents the SSR distribution in the T2 (72 h PEG treatment) sample.

\subsection{KEGG Analysis for Pathway Identification}

The KEGG database was used to identify 138 important pathways involved in various developmental processes in plants. Some of the vital pathways, such as plant-hormone signal transduction pathways, plant pathogen interaction, MAPK signaling, phenylpropanoid biosynthesis, and ubiquitin-mediated proteolysis, were mapped with a significant number of expressed transcripts (Figure 7). From the assembled transcriptome data, we could find all the expressed genes mainly involved in the MAPK pathways. In total, 38 key genes involved in the MAPK pathway were identified (Supplementary Figure S2). The number of transcripts involved in the MAPK pathway varied from 83 to 94 transcripts in different sample groups. From the expressed transcriptome, 41 enzymes were found to be involved in plant-hormone signal transduction pathways. A significant number of transcripts were mapped against each plant-hormone-mediated signal cascade process (Supplementary Figure S3).

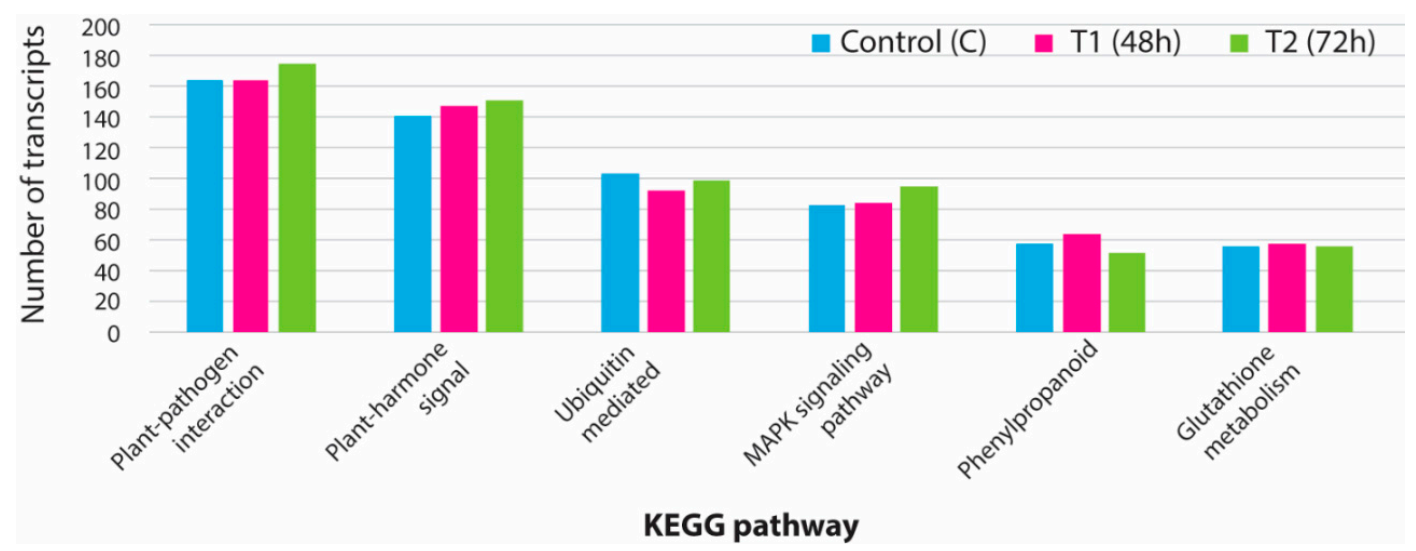

Figure 7. Total number of assembled transcripts associated with major pathways in plants mapped using Kyoto encyclopedia of genes and genomes database (KEGG). C: control; T1: 48 h PEG treatment; T2: 72 h PEG treatment. 


\subsection{In Silico Differential Gene Expression (DGE) Analysis}

Transcripts from the control, T1, and T2 samples were pooled and clustered using CD-HIT program, which resulted in 229,224 clustered transcripts. These clustered transcripts were considered as a reference for in silico DGE analysis. The read counts for all three samples were generated by aligning reads to the clustered transcripts using bowtie2, and normalized gene expression quantification was estimated using the DESeq program. Differentially expressed metabolically important enzymes were identified using KEGG database comparison (Figure 8). The DGE analysis between the control and T1 condition resulted in 1803 upregulated and 2501 downregulated transcripts ( $p$-value $<0.01$, Log2 fold change \pm 1 ). In the T2 samples, 3046 upregulated and 1582 downregulated transcripts were identified ( $p$-value $<0.01, \log 2$ fold change \pm 1 ) (Figure 9). Approximately, 1153 transcripts showed significant differential expression in both $\mathrm{T} 1$ and $\mathrm{T} 2$ samples. Functional annotation for differentially expressed transcripts was performed by aligning against the Uniprot plant protein database. The differential expression of the transcription factors expressed under different treatments were represented as heat maps (Figure 10). Compared to other TFs, the transcripts of the NAC transcription factor showed higher expression under different treatment levels.

a)

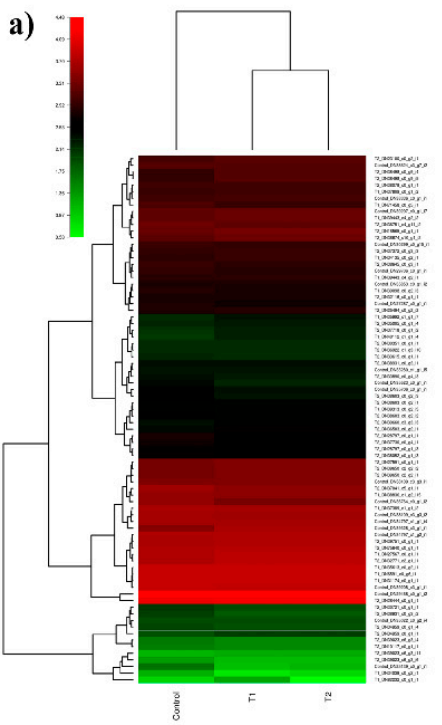

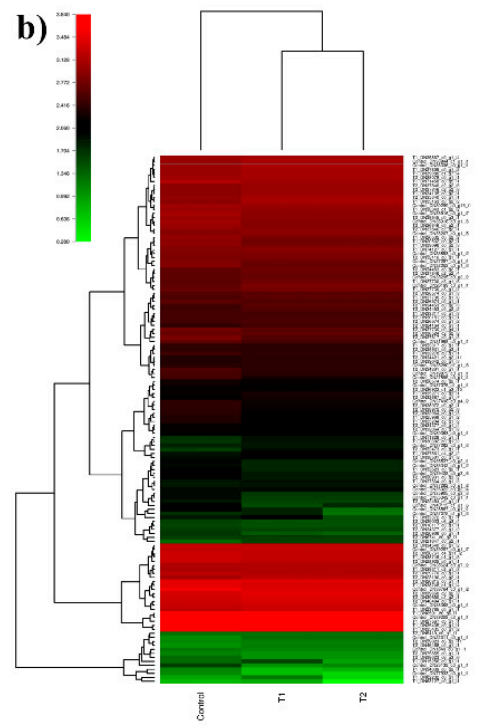

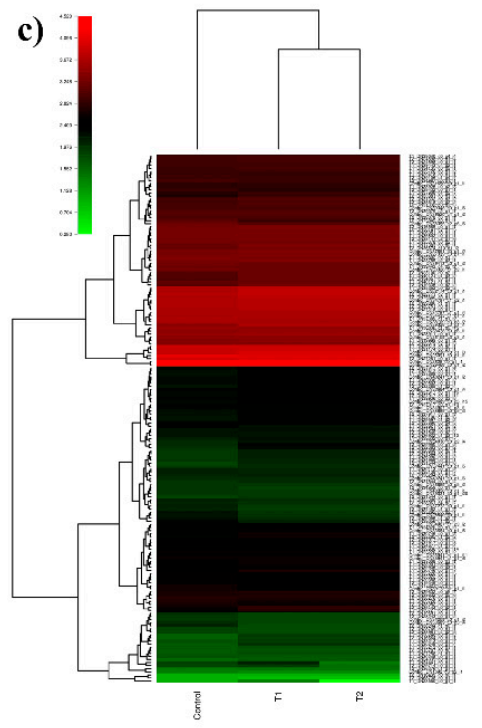

Figure 8. Differential gene expression pattern of selected KEGG pathway in H. persicum: (a) heat map showing the DGEs of MAPK-signaling genes in the control, T1 (48 h PEG treatment), and T2 (72 h PEG treatment) samples; (b) heat map showing the DGEs of plant-hormone signal transduction pathway genes in the control, T1, and T2 samples; (c) heat map showing the DGEs of plant pathogen interaction pathway genes in the control, T1, and T2 samples. 


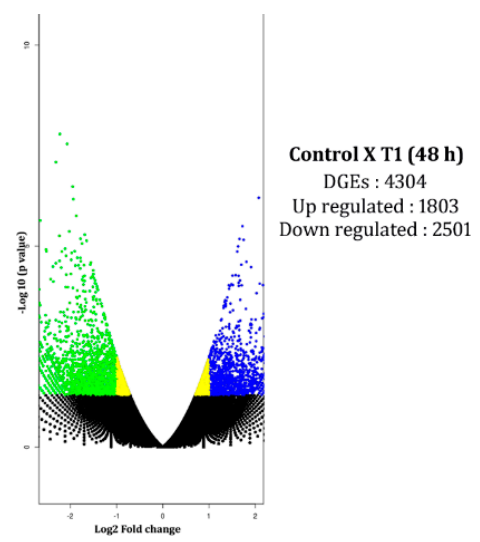

(a)

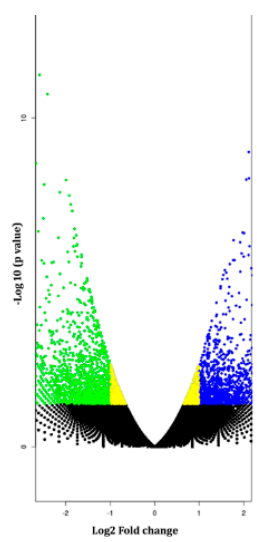

(b)

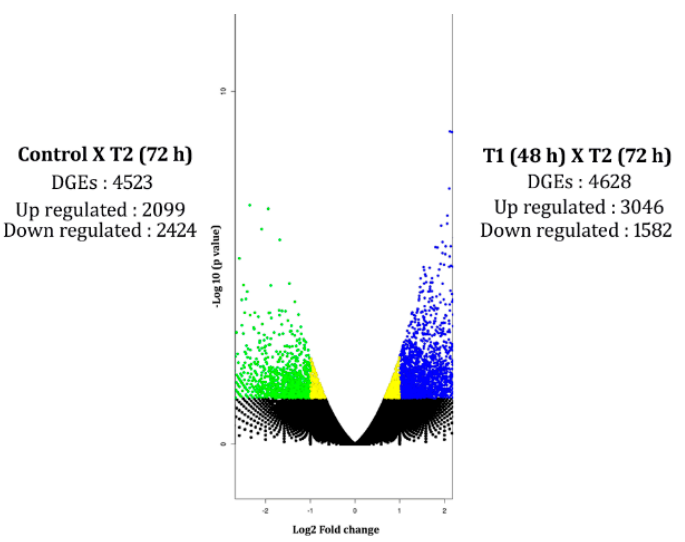

(c)

Figure 9. Volcano plots for the DEGs under different PEG treatments. Volcano plot DEGs generated for: (a) control (no drought) vs. T1 (48 h PEG treatment); (b) control (no drought) vs. T2 (72 h PEG treatment); (c) T1 (48 h PEG treatment) vs. T2 (72 h PEG treatment). The $\mathrm{X}$ and $\mathrm{Y}$ axis denote the $\log 2$-fold change and $-\log 10$ (p-value), respectively. The blue dots represent upregulated genes, green represents downregulated, black represents the neutrally regulated.

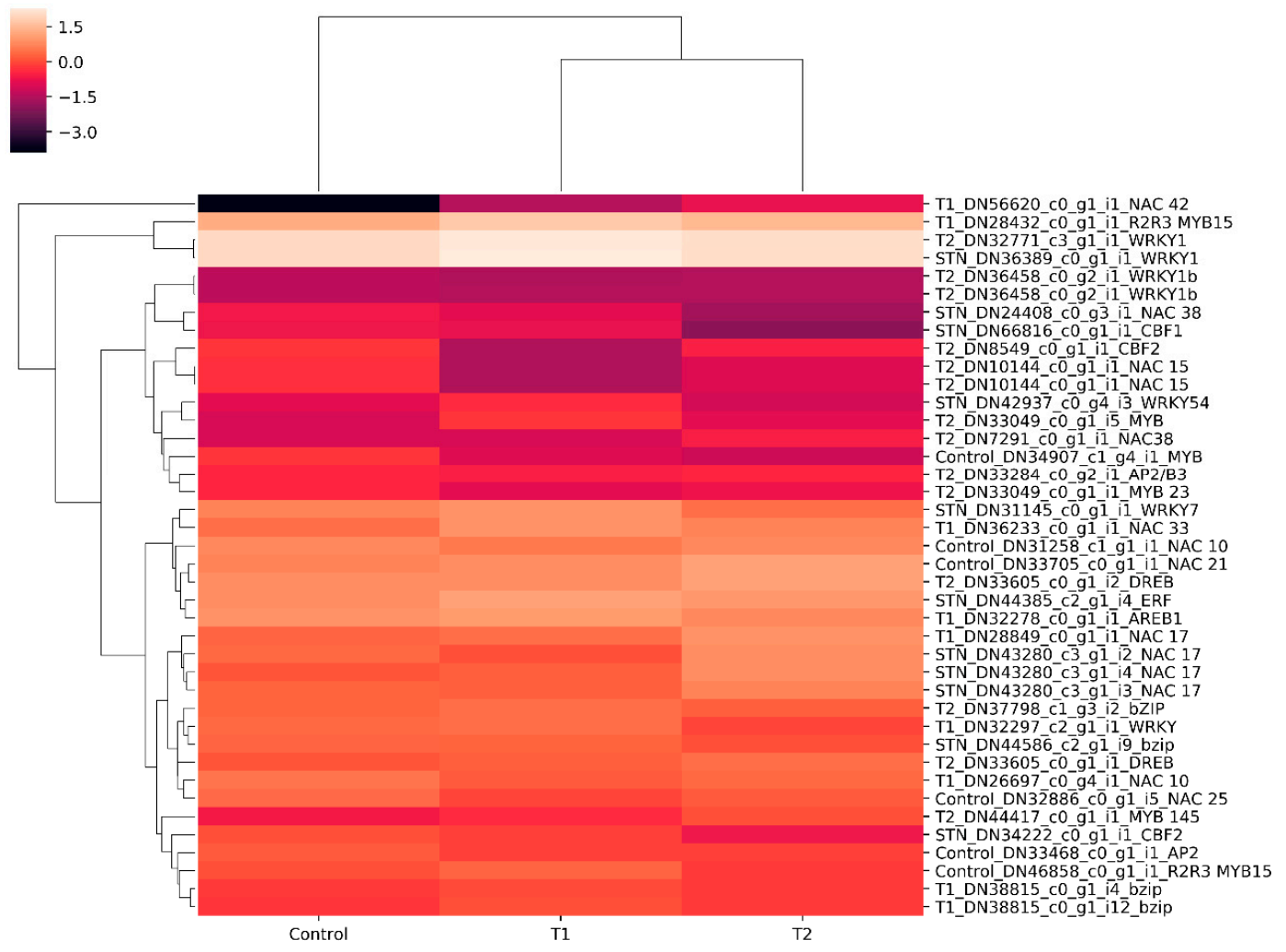

Figure 10. Differential gene expression patterns of transcription factors (TFs). Heat map showing differential expression of different TFs in the control, T1 (48 h PEG treatment), and T2 (72 h PEG treatment) samples.

\subsection{RT-PCR}

Quantitative real-time PCR analysis for the selected genes was performed to confirm the in silico DGE analysis data. From the transcript annotation, ten genes significantly expressed in both T1 (48 h PEG treatment) and T2 (72 h PEG treatment) compared to the control were selected for qPCR analysis. The analysis of ten genes, zinc finger (ZNF), cytochrome P450 (CYT), peroxidase (POX), 
glutamate decarboxylase (GAD), aldoketoreductase (AKR), calcium-transporting ATPase (CTAPSE), phosphoinositide phospholipase C (PI-PLCs), trehalase (TRE), protein phosphatase-2C (PP2C), and MYB family protein (MYB), showed positive correlations with the in silico DGE analysis data. The fold change trends in both the RNA sequence and the qPCR data are illustrated in Figure 11.

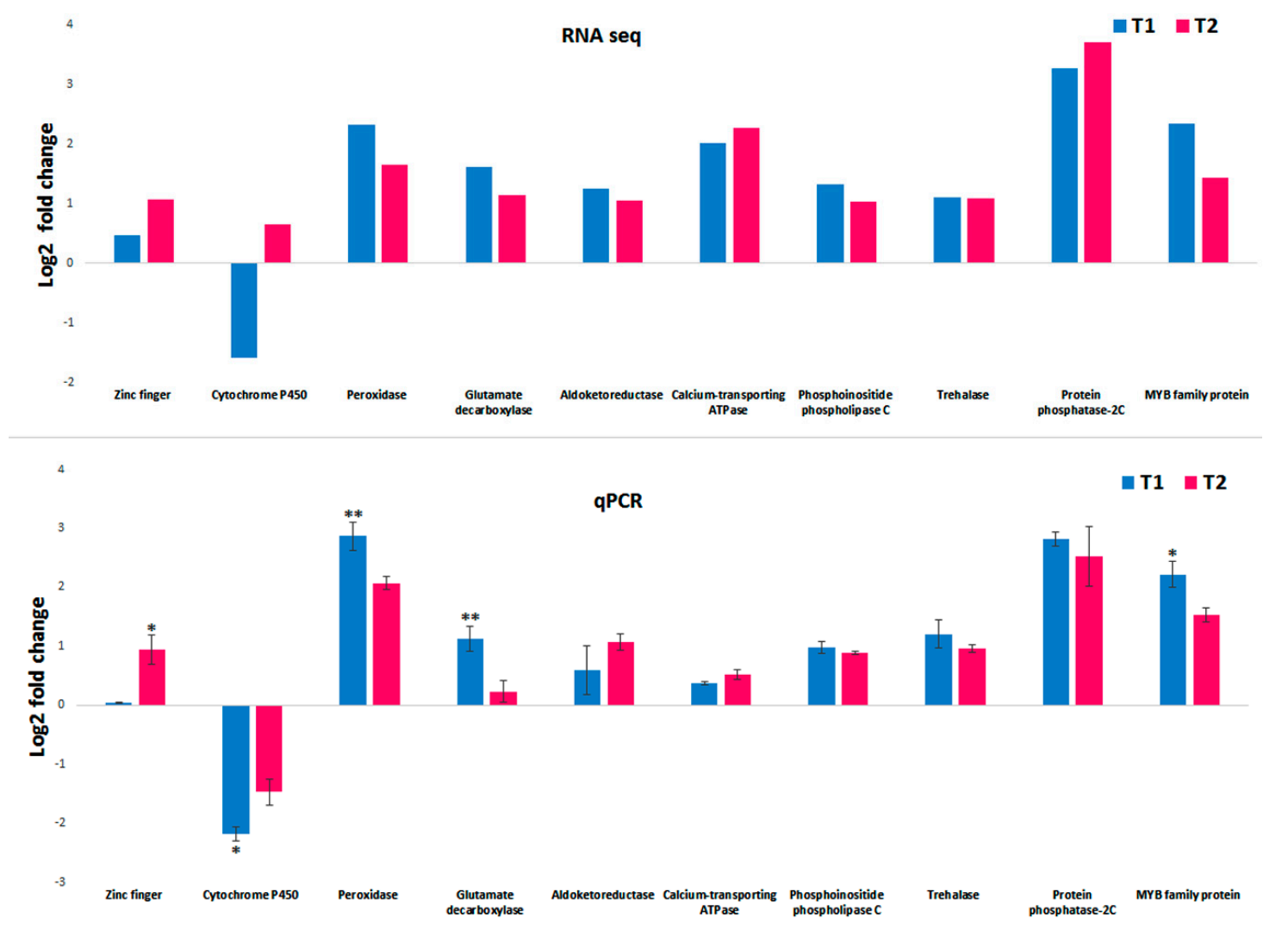

Figure 11. Validation of selected transcript from RNA seq using qPCR. The graph on the top shows the Log2FC of ten selected transcript DGE expressions in the T1 and T2 sample. The bottom graph represents the corresponding qPCR fold change in the T1 and T2 samples. The error bar represents the standard deviation. The difference between the means were calculated by Student's t-test $\left({ }^{*}: p \leq 0.05\right.$, **: $p$ 5 0.01). T1: 48 h PEG treatment; T2: 72 h PEG treatment.

\section{Discussion}

In arid regions drought, salinity and thermal stress are the major hostile environmental factors that often limit crop production. Climate change and rapid urbanization are the potential factors that cause the erosion of biodiversity leading to the extinction of many species. H. persicum, an endangered nearly extinct species native to the arid region of the Middle East, has a great potential in urban landscaping considering its ability to combat thermal and drought stress. Therefore, identifying and conserving the drought-responsive genes from such endangered species has considerable significance for enriching the gene pool and future applications in drought studies. Drought stress and oxidative stress are often interrelated and may also exhibit identical and complexly adverse effects, leading to cellular damage. Exploring the expression profile of drought-responsive genes is essential for understanding the molecular mechanisms and metabolic pathways associated with drought-stress tolerance in crops [6,32].

The focus of the present study was to identify the upregulated and downregulated genes involved in the growth and maintenance of adventitious shoot buds developed in vitro under PEG-induced drought. PEG $6000\left(100 \mathrm{~g} \mathrm{~L}^{-1}\right)$ was used in the media to create a rapid drought stress through water deprivation [33]. A significant decline in fresh weight, dry weight, RWC, and tissue water potential (Mpa) along with changes in the phenology of the adventitious shoot buds confirmed the PEG-induced 
drought stress. Transcriptional reprogramming under stress is one of the major mechanisms that plants undergo during stress tolerance. In the present study, transcriptome profiles of the two drought-induced (PEG) samples T1 (48 h) and T2 (72 h) were compared with the control samples without any drought treatment. This resulted in 82,440 assembled transcript (T1) with an average length of $978 \mathrm{bp}$ (control). The N50 lengths were 1437, 1467, and 1524 bp for the control, T1, and T2, respectively. These findings are comparable with the results of RNA-sequence in Haloxylon ammodendron (N50: $1345 \mathrm{bp}$ ) under drought stress [34].

During drought stress, significant changes in gene expression occur in plants in order to survive under drought by regulating cellular process [35]. Elucidating the drought-stress tolerance in plants by differential gene-expression methods provides valuable information for identifying probable stress tolerance mechanism. The DGE analysis displayed the differential expression of approximately 1153 transcripts in both T1 and T2 samples, which was relatively higher in number compared to H. ammodendron transcriptome [34]. In the present study, the number of DEGs showed a significant change when the PEG stress was increased from $48 \mathrm{~h}$ to $72 \mathrm{~h}$. Similar findings were reported in cassava, where a significant changes observed in DEGs when the PEG stress period was increased from $3 \mathrm{~h}$ to $24 \mathrm{~h}$ [36]. In addition, our results indicate that the differential expression of downregulated genes showed gradual reduction in number upon increase in the PEG stress period, whereas the upregulated genes showed a steady increase in number under elevated stress period. Overall, the percentage of downregulated genes was higher in both treatment conditions (Figure 9). These results were in alignment with the findings of Liu et al. (2014), and Ma et al. (2015), who reported that the abundance of downregulated DGEs was significantly higher compared to upregulated genes under PEG stress [16,17].

The transcription factors are regulatory proteins which play a major role in drought tolerance by synchronizing the signaling network and gene expression under stress [37]. NAC transcription factors are ubiquitous in plants, and their expression shows significant changes under abiotic stresses [38]. In our dataset, the expression of the NAC transcription factor (DN33705_c0_g1_i1) gene was upregulated under PEG stress, compared to the control (Figure 10). The expression level elevated significantly when the PEG treatment period shifted from $48 \mathrm{~h}$ to $72 \mathrm{~h}$. The involvement of the NAC TFs in drought tolerance has been demonstrated in various crops like rice [39], and soybean [40]. Dehydration responsive element binding factor (DREB) is an AP2/ERF-type transcription factor present in plants [41]. The overexpression of the OsDREB2B gene in Arabidopsis resulted in improved tolerance to drought stress [42]. In the present study, DREB (DN33605_c0_g1_i2) transcripts displayed significant upregulation under PEG-induced stress; this denotes the probable role of DREB TFs in the downstream process of the drought-tolerance mechanism in H. persicum. Other drought-responsive TFs, such as MYB, WRKY, bZIP, bHLH, and C2H2, showed differential expression under PEG-induced drought stress, as reported in other plant species [43].

The GO enrichment analysis was performed to predict the role of transcripts in the biological, cellular, and molecular process of the cells. In all the three sample groups, more than $50 \%$ of the DEGs were involved in various GO terms. Drought-induced genes are functionally classified as two major groups, namely functional proteins and regulatory proteins. Functional proteins are involved in stress tolerance and the regulatory proteins have a major role in stress response through alteration gene expression and signal transduction [7]. The KEGG pathway analysis resulted in mapping the DEGs with 138 different pathways that were involved in various cellular processes. The MAPK cascades are a multigene family of regulatory networks deployed for the transduction of intra- and extra-cellular signals to the nucleus for suitable adjustments of the cell to the stimuli [13]. In this study, 38 potential genes were mapped with the MAPK pathway, which includes the expression of all the three components of MAPK cascade (MAPKKK, MAPKK and MAPK). MAP kinases that are involved in ethylene, jasmonic acid, and abscisic acid-mediated stress responses are significantly mapped in the present study (Supplementary Figure S2). 
Phytohormones play a significant role in plant stress regulation. During drought induction, the environmental stress factors induce the secretion of plant hormones which mediates the immediate cell responses by triggering plant hormone signal transduction pathways. Studies show that abscisic acid exhibit an important role in the drought tolerance of plants through stomatal closure and the activation of the stress-responsive genes [7]. In the present study, under PEG-induced stress, the ABA-mediated drought response facilitated by PP2C gene (Transcript Id: T2_DN28928_c0_g1_i1) was mapped, which in turn triggered the ABF (ABA-responsive element binding factor) (DN22654_c0_g1_i1) gene, which plays a major role in stomatal closure (Figure 12). Similar responses were reported in Phormium tenax [44]. Reports suggests that, the overexpression of abscisic acid stress ripening (ASR) gene family play a significant role in drought and salinity stress [45]. We found upregulation of ASR gene (DN23961_c0_g2_i3) was observed under PEG induced drought stress, which indicates the possible role of ASR genes in ABA-mediated stress tolerance. Increased stress tolerance in response to the overexpression of ASR genes was reported in transgenic plants under various stress conditions [46-49].

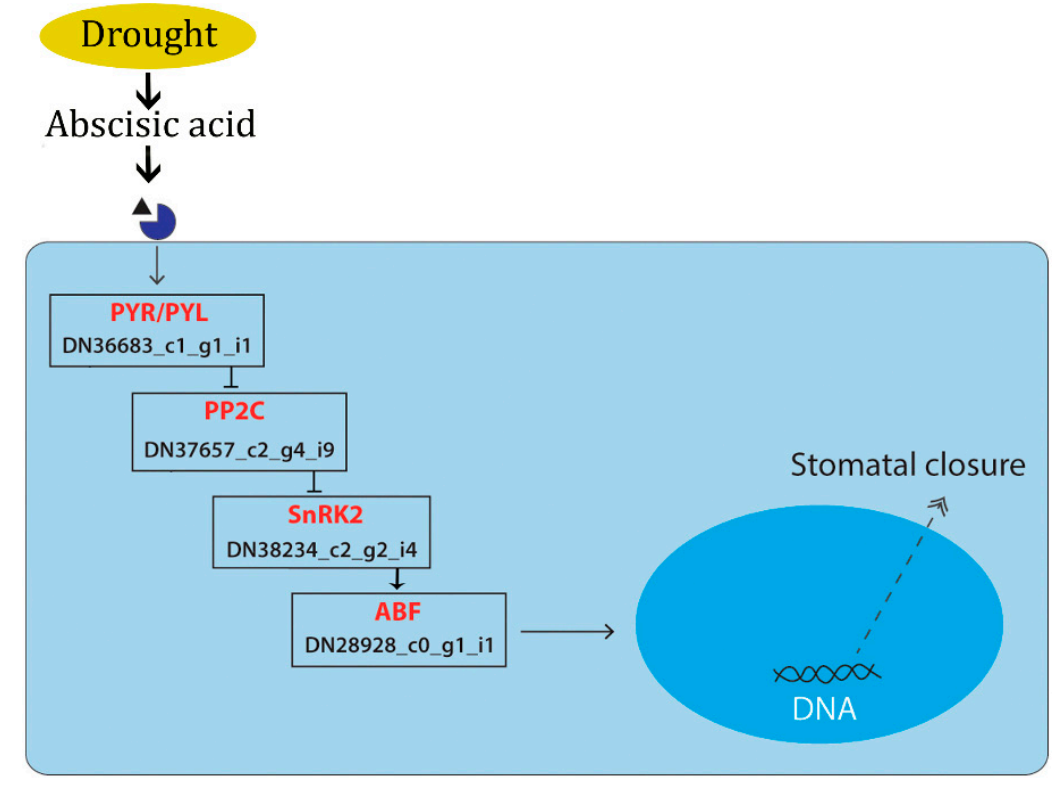

Figure 12. Schematic representation showing the transcript expressed under PEG-induced drought stress mapped in the ABA-mediated KEGG pathway. The genes expressed in the present study are given in red font and the transcript ID is given in black font.

In the present study, the ethylene-mediated plant-hormone signal transduction pathway was also explored. Ethylene is an important plant-growth regulator which regulates the plant growth under drought condition by undertaking various developmental changes in the plant [50]. Mapping the DEG for ethylene-responsive transcription factor (ERF) 1 in the KEGG pathway denotes the possible activation of ethylene-induced defense mechanism under PEG-induced stress conditions. The role of ERFs in the developmental process such as cuticle biosynthesis was reported earlier in Arabidopsis [51]. The crosslink between the jasmonic acid and ethylene-mediated pathway observed in the MAPK-signaling cascade, shows the possible role of jasmonic acid signaling intermediates in ethylene-induced defense response (Supplementary Figure S2). These findings are in accordance with the observation of Lorenzo et al. (2002), who reported that the ERF1 expression could be regulated by ethylene and jasmonic acid or the synergistic effect of both hormones [52].

The accumulation of reactive oxygen species (ROS) is one of the major modifications to occur under drought or salt stress, causing damages to the cell membranes [53]. Antioxidant enzymes play a crucial role in drought-defense mechanisms by scavenging ROS generated under drought 
stress [54]. Peroxidase is an important antioxidant enzyme involved in the detoxification of $\mathrm{H}_{2} \mathrm{O}_{2}$ molecules generated under stress. In our study, 30 DEGs matching the expression of peroxidase (EC 1.11.1.7) enzyme were identified. The expression of the enzyme was greater in the T2 sample $(72 \mathrm{~h})$ compared to the T1 $(48 \mathrm{~h})$ treatment. This shows the increased accumulation of peroxidase enzyme with a concomitant increase in PEG-induced drought stress. A similar pattern of expression changes in genes associated with peroxidases have been reported in many different plant species under drought stress [55]. Several other antioxidant enzymes, such as superoxide dismutase (SOD), catalase (CAT) and glutathione s-transferase (GST), displayed an upregulated DEG profile, which confirms the biochemical rearrangements in $H$. persicum under the PEG-induced drought stress condition.

\section{Conclusions}

In this study, we investigated the transcriptome profile of $H$. persicum, an endangered desert plant species. This is the first report on this plant, and we reported $\sim 50,000$ annotated transcripts and their possible metabolic pathways under PEG-induced drought stress. In this analysis, we identified the expression pattern of drought-responsive genes using differential gene expression analysis, which resulted in a minimum of 4304 DEGs under $48 \mathrm{~h}$ PEG treatment and a maximum of 4523 DEGs under $72 \mathrm{~h}$ PEG treatment. Elucidating the pathway results revealed the possible defense mechanism under PEG-induced drought in H. persicum. The present study will provide a better understanding of drought-response mechanism of the desert plant under stress conditions. Some differences may occur between the responses to PEG-induced drought and the drought under field conditions; nevertheless this provides valuable evidence to confirm the transcriptional reprogramming of genes under stress.

Supplementary Materials: The following are available online at http://www.mdpi.com/2073-4425/11/6/640/s1, Supplementary Table S1: List of primers used for qPCR validation; Table S2: Transcriptome annotation file for control sample; Table S3: Transcriptome annotation file for T1 sample (48h of PEG stress); Table S4: Transcriptome annotation file for T2 sample (72h of PEG stress); Figure S1: Percentage of ATGC content in H. persicum. (a) ATGC content in control sample, (b) ATGC content in T1 sample (c) ATGC content in T2 sample; Figure S2: KEGG pathway genes mapped for MAPK signaling in H. persicum under PEG induced stress (Red box denotes genes expressed in the present study); Figure S3: KEGG pathway genes mapped for plant hormone signal transduction in H. persicum under PEG induced stress. (Red box denotes genes expressed in the present study).

Author Contributions: S.S.K. and K.M.A.A. conceived the study and designed the experiment. F.T.P. and N.H.T. performed the methodology section. B.R. and F.T.P. performed the bioinformatics analyses and prepared figures and texts for the manuscript. S.S.K., B.R. and F.T.P. wrote the first draft of the manuscript. K.M.A.A., A.J.C. and S.S. participated in the discussion part, S.S.K. and B.R. revised the manuscript. All authors have read and agreed to the published version of the manuscript.

Funding: This study was supported by grants from Khalifa Center for Genetic Engineering and Biotechnology (31R004), UAE University.

Acknowledgments: This study was supported by the grant received from Khalifa Center for Genetic Engineering and Biotechnology, UAE University, which is greatly acknowledged. The authors would like to thank the Dean, College of Food and Agriculture, UAE University for extending research infrastructure and Ghaleb Ali Al Hadrami, Acting Vice Chancellor/Deputy Vice Chancellor for Academic Affairs and Provost, UAE University for his help and support.

Conflicts of Interest: The authors declare that they have no conflict of interests.

Data availability: The datasets generated are available in NCBI-SRA database with samples IDs for control SAMN08281247, T1 sample SAMN08281244, and T2 sample SAMN08281248.

\section{References}

1. Hadi, M.; Kharazipour, R. The effect of various moisture treatments on water use efficiency (WUE) in Haloxylon plant. In Review of Forests, Wood Products and Wood Biotechnology of Iran and Germany-Part II; Schöpper, A.R., Müller, C., Eds.; Universitätsverlag: Göttingen, Germany, 2008.

2. Zohary, M. Geobotanical foundations of the Middle East; Gustav Fischer Verlag: Stuttgart, Germany, 1973; Volume 1. 
3. Lawson, T.; Oxborough, K.; Morison, J.I.L.; Baker, N.R. The responses of guard and mesophyll cell photosynthesis to $\mathrm{CO}_{2}, \mathrm{O}_{2}$, light and water stress in a range of species are similar. J. Exp. Bot. 2003, 54, 1743-1752. [CrossRef] [PubMed]

4. Kiani, S.P.; Grieu, P.; Maury, P.; Hewezi, T.; Gentzbittel, L.; Sarrafi, A. Genetic variability for physiological traits under drought conditions and differential expression of water stress-associated genes in sunflower (Helianthus annuus L.). Theor. Appl. Genet. 2007, 114, 193-207. [CrossRef] [PubMed]

5. Ramanjulu, S.; Bartels, D. Drought and desiccation induced modulation of gene expression in plants. Plant Cell Environ. 2002, 25, 141-151. [CrossRef] [PubMed]

6. Valliyodan, B.; Nguyen, H.T. Understanding regulatory networks and engineering for enhanced drought tolerance in plants. Curr. Opin. Plant Biol. 2006, 9, 189-195. [CrossRef] [PubMed]

7. Shinozaki, K.; Yamaguchi-Shinozaki, K. Gene networks involved in drought stress tolerance and response. J. Exp. Bot. 2007, 58, 221-227. [CrossRef] [PubMed]

8. Golldack, D.; Li, C.; Mohan, H.; Probst, N. Tolerance to drought and salt stress in plants: Unravelling the signaling networks. Front Plant Sci. 2014, 5, 151. [CrossRef] [PubMed]

9. Raghavendra, A.S.; Gonugunta, V.K.; Christmann, A.; Grill, E. ABA perception and signalling. Trends Plant Sci. 2010, 15, 395-401. [CrossRef] [PubMed]

10. Kumar, M.; Kesawat, M.S.; Ali, A.; Lee, S.C.; Gill, S.S.; Kim, A.H.U. Integration of abscisic acid signaling with other signaling pathways in plant stress responses and development. Plants 2019, 8, 592. [CrossRef] [PubMed]

11. Akpinar, B.A.; Avsar, B.; Lucas, S.J.; Budak, H. Plant abiotic stress signaling. Plant Signal. Behav. 2012, 7, 1450-1455. [CrossRef] [PubMed]

12. Yu, W.; Zhao, R.; Wang, L.; Zhang, S.; Li, R.; Sheng, J.; Shen, L. ABA signaling rather than ABA metabolism is involved in trehalose-induced drought tolerance in tomato plants. Planta 2019, 250, 643-655. [CrossRef] [PubMed]

13. Sinha, A.K.; Monika, J.; Badmi, R.; Narendra, T. Mitogen-activated protein kinase signaling in plants under abiotic stress. Plant Signal. Behav. 2011, 6, 196-203. [CrossRef] [PubMed]

14. Bigeard, J.; Hirt, H. Nuclear Signaling of Plant MAPKs. Front. Plant Sci. 2018, 9, 469. [CrossRef] [PubMed]

15. Mahmood, T.; Khalid, S.; Abdullah, M.; Ahmed, Z.; Shah, M.K.N.; Ghafoor, A.; Du, X. Insights into Drought Stress Signaling in Plants and the Molecular Genetic Basis of Cotton Drought Tolerance. Cells 2020, 9, 105. [CrossRef]

16. Miller, G.; Suzuki, N.; Ciftci-Yilmaz, S.; Mittler, R. Reactive oxygen species homeostasis and signalling during drought and salinity stresses. Plant Cell Environ. 2010, 33, 453-467. [CrossRef] [PubMed]

17. Malinich, E.A.; Wang, K.; Mukherjee, P.K.; Kolomiets, M.; Kenerley, C.M. Differential expression analysis of Trichoderma virens RNA reveals a dynamic transcriptome during colonization of Zea mays roots. BMC Genom. 2019, 20, 280. [CrossRef] [PubMed]

18. Kumar, M.; Kim, I.; Kim, Y.K.; Heo, J.B.; Suh, M.C.; Kim, H.U. Strigolactone Signaling Genes Showing Differential Expression Patterns in Arabidopsis max Mutants. Plants 2019, 8, 352. [CrossRef] [PubMed]

19. Zhua, J.; Xub, Q.; Zhaoa, S.; Xiaa, X.; Yana, X.; Ana, Y.; Mia, X.; Guoa, L.; Samarinac, L.; Wei, C. Comprehensive co-expression analysis provides novel insights into temporal variation of flavonoids in fresh leaves of the tea plant (Camellia sinensis). Plant Sci. 2020, 290, 110306. [CrossRef] [PubMed]

20. Liu, Y.; Liu, M.; Li, X.; Cao, B.; Ma, X. Identification of Differentially Expressed Genes in Leaf of Reaumuria soongorica under PEG-Induced Drought Stress by Digital Gene Expression Profiling. PLoS ONE 2014, 9, e94277. [CrossRef] [PubMed]

21. Ma, X.; Wang, P.; Zhou, S.; Sun, Y.; Liu, N.; Li, X.; Hou, Y. De novo transcriptome sequencing and comprehensive analysis of the drought-responsive genes in the desert plant Cynanchum komarovii. BMC Genom. 2015, 16, 753. [CrossRef] [PubMed]

22. Barrs, H.D.; Weatherley, P.E. A re-examinati on of the relative turgidity technique for estimating water deficit in leaves. Aust. J. Biol. Sci. 1962, 15, 413-428. [CrossRef]

23. Andrews, S. FastQC: A Quality Control Tool for High Throughput Sequence Data. 2010. Available online: https://www.bioinformatics.babraham.ac.uk/projects/fastqc/ (accessed on 13 January 2020).

24. Grabherr, M.G.; Haas, B.J.; Yassour, M.; Levin, J.Z.; Thompson, D.A.; Amit, I.; Adiconis, X.; Fan, L.; Raychowdhury, R.; Zeng, Q.; et al. Full-length transcriptome assembly from RNA-seq data without a reference genome. Nat. Biotechnol. 2011, 29, 644-652. [CrossRef] [PubMed] 
25. Altschul, S.F.; Madden, T.L.; Schäffer, A.A.; Zhang, J.; Zhang, Z.; Miller, W.; Lipman, D.J. Gapped BLAST and PSI-BLAST: A new generation of protein database search programs. Nucleic Acids Res. 1997, 25, 3389-3402. [CrossRef] [PubMed]

26. Conesa, A.; Götz, S.; García-Gómez, J.M.; Terol, J.; Talón, M.; Robles, M. Blast2GO: A universal tool for annotation, visualization and analysis in functional genomics research. Bioinformatics 2005, 21, 3674-3676. [CrossRef] [PubMed]

27. Tatusov, R.L.; Fedorova, N.D.; Jackson, J.D.; Jacobs, A.R.; Kiryutin, B.; Koonin, E.V.; Krylov, D.M.; Mazumder, R.; Mekhedov, S.L.; Nikolskaya, A.N.; et al. The COG database: An updated version includes eukaryotes. BMC Bioinform. 2003, 4, 41. [CrossRef] [PubMed]

28. Moriya, Y.; Itoh, M.; Okuda, S.; Yoshizawa, A.C.; Kanehisa, M. KAAS: An automatic genome annotation and pathway reconstruction server. Nucleic Acids Res. 2007, 35, W182-W185. [CrossRef] [PubMed]

29. Kanehisa, M.; Susumu, G. KEGG: Kyoto encyclopedia of genes and genomes. Nucleic Acids Res. 2000, 28, 27-30. [CrossRef] [PubMed]

30. Langmead, B.; Steven, L.S. Fast gapped-read alignment with Bowtie 2. Nat. Methods 2012, 9, 357-359. [CrossRef] [PubMed]

31. Anders, S.; Wolfgang, H. Differential Expression of RNA-Seq Data at the Gene Level-the DESeq Package; European Molecular Biology Laboratory (EMBL): Heidelberg, Germany, 2012.

32. Mayra, R.; Eduardo, C.; Orlando, B. Molecular aspects of abiotic stress in plants. Biotecnol. Appl. 2005, 22, 10.

33. Caruso, A.; Chefdor, F.; Carpin, S.; Depierreux, C.; Delmotte, F.M.; Kahlem, G.; Morabito, D. Physiological characterization and identification of genes differentially expressed in response to drought induced by PEG 6000 in Populus canadensis leaves. J. Plant Physiol. 2008, 165, 932-941. [CrossRef] [PubMed]

34. Long, Y.; Zhang, J.; Tian, X.; Wu, S.; Zhang, Q.; Zhang, J.; Dang, Z.; Pei, X.W. De novo assembly of the desert tree Haloxylon ammodendron (CA Mey.) based on RNA-Seq data provides insight into drought response, gene discovery and marker identification. BMC Genom. 2014, 15, 1111. [CrossRef] [PubMed]

35. Shanker, A.K.; Maheswari, M.; Yadav, S.K.; Desai, S.; Bhanu, D.; Attal, N.B.; Venkateswarlu, B. Drought stress responses in crops. Funct. Integr. Genom. 2014, 14, 11. [CrossRef] [PubMed]

36. Fu, L.; Ding, Z.; Han, B.; Hu, W.; Li, Y.; Zhang, J. Physiological investigation and transcriptome analysis of polyethylene glycol (PEG)-induced dehydration stress in Cassava. Int. J. Mol. Sci. 2016, 17, 283. [CrossRef] [PubMed]

37. Joshi, R.; Wani, S.H.; Singh, B.; Bohra, A.; Dar, Z.A.; Lone, A.A.; Pareek, A.; Singla-Pareek, S.L. Transcription factors and plants response to drought stress: Current understanding and future directions. Front Plant Sci. 2016, 7, 1029. [CrossRef] [PubMed]

38. Nakashima, K.; Takasaki, H.; Mizoi, J.; Shinozaki, K.; Yamaguchi-Shinozaki, K. NAC transcription factors in plant abiotic stress responses. Biochim. Biophys. Acta 2012, 1819, 97-103. [CrossRef] [PubMed]

39. Shao, H.; Wang, H.; Tang, X. NAC transcription factors in plant multiple abiotic stress responses: Progress and prospects. Front Plant Sci. 2015, 6, 902. [CrossRef] [PubMed]

40. Le, D.T.; Nishiyama, R.; Watanabe, Y.; Mochida, K.; Yamaguchi-Shinozaki, K.; Shinozaki, K.; Tran, L.S.P. Genome-wide survey and expression analysis of the plant-specific NAC transcription factor family in soybean during development and dehydration stress. DNA Res. 2011, 18, 263-276. [CrossRef] [PubMed]

41. Sharoni, A.M.; Nuruzzaman, M.; Satoh, K.; Shimizu, T.; Kondoh, H.; Sasaya, T.; Choi, I.R.; Omura, T.; Kikuchi, S. Gene structures, classification and expression models of the AP2/EREBP transcription factor family in rice. Plant Cell Physiol. 2011, 52, 344-360. [CrossRef] [PubMed]

42. Matsukura, S.; Mizoi, J.; Yoshida, T.; Todaka, D.; Ito, Y.; Maruyama, K.; Shinozaki, K.; Yamaguchi-Shinozaki, K. Comprehensive analysis of rice DREB2-type genes that encode transcription factors involved in the expression of abiotic stress-responsive genes. Mol. Genet. Genom. 2010, 283, 185-196. [CrossRef] [PubMed]

43. Yang, Z.; Dai, Z.; Lu, R.; Wu, B.; Tang, Q.; Xu, Y.; Cheng, C.; Su, J. Transcriptome Analysis of Two Species of Jute in Response to Polyethylene Glycol (PEG)-induced Drought Stress. Sci. Rep. 2017, 7, 16565. [CrossRef] [PubMed]

44. Bai, Z.; Wang, T.; Wu, Y.; Wang, K.; Liang, Q.; Pan, Y.; Jiang, B.; Zhang, L.; Liu, G.; Jia, Y.; et al. Whole-transcriptome sequence analysis of differentially expressed genes in Phormium tenax under drought stress. Sci. Rep. 2017, 7, 41700. [CrossRef] [PubMed]

45. Golan, I.; Dominguez, P.G.; Konrad, Z.; Shkolnik-Inbar, D.; Carrari, F.; Bar-Zvi, D. Tomato abscisic acid stress ripening (ASR) gene family revisited. PLoS ONE 2014, 9, e107117. [CrossRef] [PubMed] 
46. Yang, C.Y.; Chen, Y.C.; Jauh, G.Y.; Wang, C.S. A lily ASR protein involves abscisic acid signaling and confers drought and salt resistance in Arabidopsis. Plant Physiol. 2005, 139, 836-846. [CrossRef] [PubMed]

47. Dai, J.R.; Liu, B.; Feng, D.R.; Liu, H.; He, Y.; Qi, K.B.; Wang, H.B.; Wang, J.F. MpAsr encodes an intrinsically unstructured protein and enhances osmotic tolerance in transgenic Arabidopsis. Plant Cell Rep. 2011, 30, 1219-1230. [CrossRef] [PubMed]

48. Hsu, Y.F.; Yu, S.C.; Yang, C.Y.; Wang, C.S. Lily ASR protein-conferred cold and freezing resistance in Arabidopsis. Plant Physiol. Biochem. 2011, 49, 937-945. [CrossRef] [PubMed]

49. Jha, B.; Lal, S.; Tiwari, V.; Yadav, S.K.; Agarwal, P.K. The SbASR-1 gene cloned from an extreme halophyte Salicornia brachiata enhances salt tolerance in transgenic tobacco. Mar. Biotechnol. 2012, 14, 782-792. [CrossRef] [PubMed]

50. Ecker, J.R. The ethylene signal transduction pathway in plants. Science 1995, 268, 667-675. [CrossRef] [PubMed]

51. Borisjuk, N.; Hrmova, M.; Lopato, S. Transcriptional regulation of cuticle biosynthesis. Biotechnol. Adv. 2014, 32, 526-540. [CrossRef] [PubMed]

52. Lorenzo, O.; Piqueras, R.; Sánchez-Serrano, J.J.; Solano, R. Ethylene response factor 1 integrates signal from ethylene and jasmonate pathway in plant defense. Plant Cell. 2002, 15, 165-178. [CrossRef] [PubMed]

53. Zhu, J.; Jianping, N.I. Abiotic Stress Signalling and Responses in Plants. Cell 2016, 167, 313. [CrossRef] [PubMed]

54. Fini, A.; Guidi, L.; Ferrini, F.; Brunettia, C.; Di Ferdinando, M.; Biricolti, S.; Pollastri, S.; Calamaia, L.; Tattini, M. Drought stress has contrasting effects on antioxidant enzymes activity and phenylpropanoid biosynthesis in Fraxinusornus leaves: An excess light stress affair? J. Plant. Physiol. 2012, 169, 929-939. [CrossRef] [PubMed]

55. Baldoni, E.; Monica, M.; Franca, L.; Roberto, C.; Laura, R.C.; Valentina, P.; Pamela, A.; Annamaria, G. Analysis of transcript and metabolite levels in Italian rice (Oryza sativa L.) cultivars subjected to osmotic stress or benzothiadiazole treatment. Plant Physiol. Biochem. 2013, 70, 492e503. [CrossRef] [PubMed]

(C) 2020 by the authors. Licensee MDPI, Basel, Switzerland. This article is an open access article distributed under the terms and conditions of the Creative Commons Attribution (CC BY) license (http://creativecommons.org/licenses/by/4.0/). 